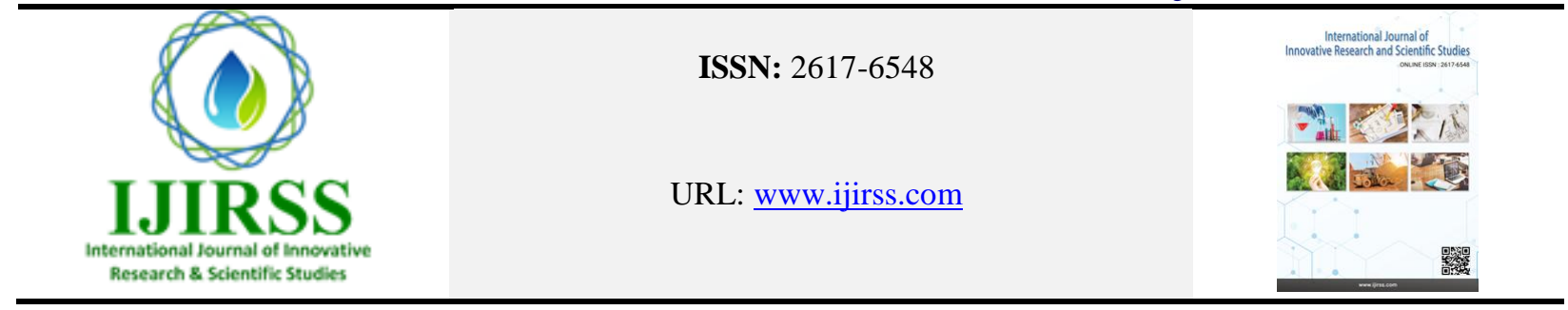

\title{
Stabilization of Crude Oil Emulsions Using Different Surfactants
}

\author{
Sweeta Akbari $^{1 *}$, Abdurahman Hamid Nour ${ }^{2}$ \\ 1,2 Faculty of Chemical \& Natural Resources Engineering, Universiti Malaysia Pahang, Lebuhraya Tun Razak, 26300 Gambang, \\ Pahang, Malaysia \\ *Corresponding author: Sweeta Akbari (sweeta.akbary11@yahoo.com)
}

\begin{abstract}
Emulsions can be found in different industries such as petroleum, food, cosmetic and pharmaceutics. Generally, there are two types of emulsions in petroleum industries: water-in-oil (W/O) and oil-in-water $(\mathrm{O} / \mathrm{W})$. The aim of this research was to evaluate the stability of W/O emulsions using different emulsifiers such as Span 80, Span 83, Triton-x-100, DEA, and LSWR with different concentrations (1.5 and 2.5) vol\%. All the emulsions were prepared at (20-80) vol\% and $2000 \mathrm{rpm}$. The result of this study showed that the most stable emulsions were prepared by Span 80. However, LSWR formed the most unstable emulsions. In addition, it was also found that the concentration of emulsifier can significantly affect the emulsion stability.
\end{abstract}

Keywords: Emulsion, Surfactant, Droplet size, Span 80.

DOI: 10.53894 /ijirss.v1i1.6

Funding: This study received no specific financial support.

History: Received: 25 February 2018/Revised: 8 March 2018/Accepted: 19 March 2018/Published: 21 September 2018

Licensed: This work is licensed under a Creative Commons Attribution 4.0 License $(\mathrm{cc})$ EY

Acknowledgement: Both authors contributed to the conception and design of the study.

Competing Interests: The authors declare that they have no conflict of interests.

Transparency: The authors confirm that the manuscript is an honest, accurate, and transparent account of the study was reported; that no vital features of the study have been omitted; and that any discrepancies from the study as planned have been explained.

Ethical: This study follows all ethical practices during writing. 


\title{
تثبيت امولسيون هاى نفت خام با استفاده از سورفاكتاتت هاى مختلف
}

\author{
"سويتا اكبرى، عبدالرحمن حامد نور \\ فاكولته /نجينيرى كييلاوى و منابع طبيعى، بوهنتون بهنحى ماليزيا، كوانتن، بهنح، ماليزيا
}

\section{خلاصه}

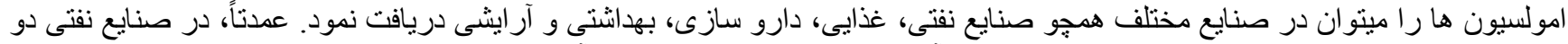

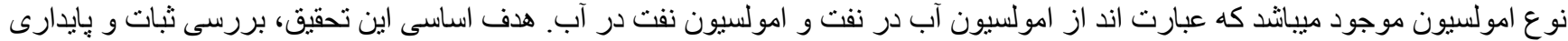

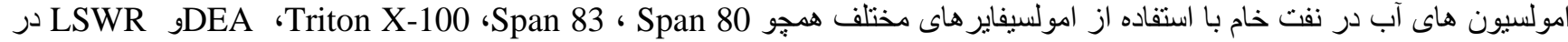

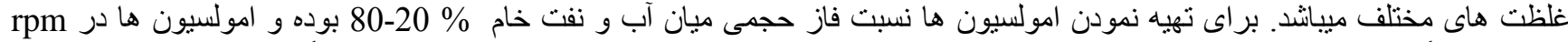

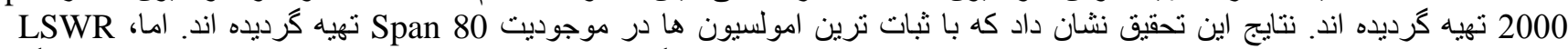

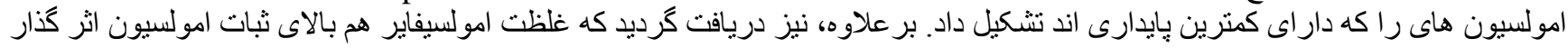

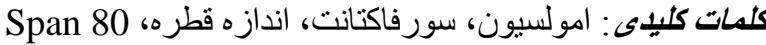

1

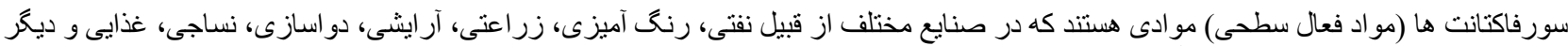

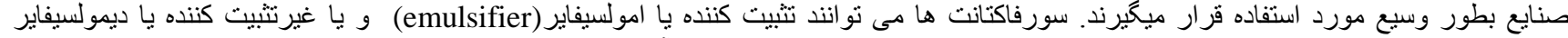
(demulsifier)

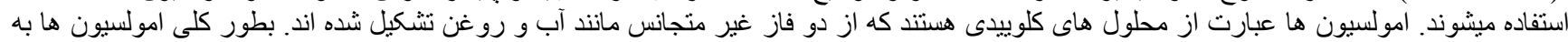

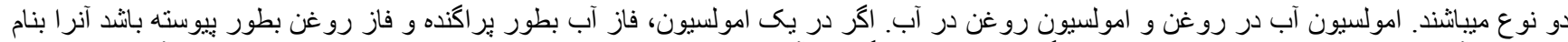

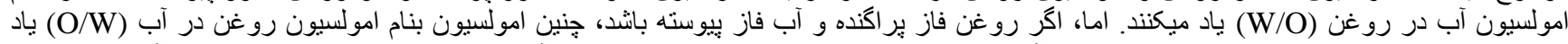

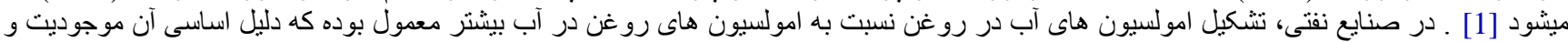

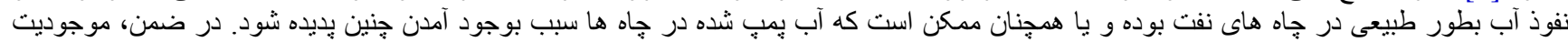

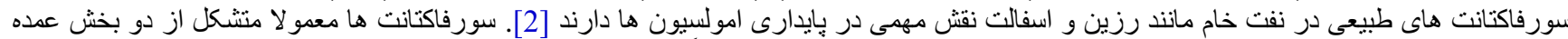

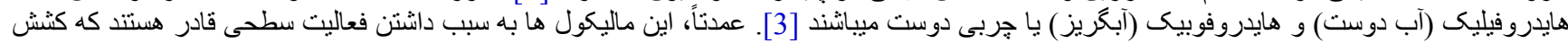

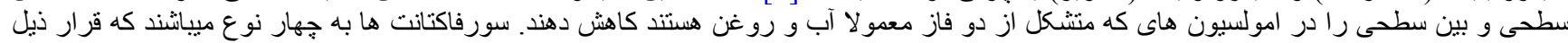

توضيح داده شده اند [4].

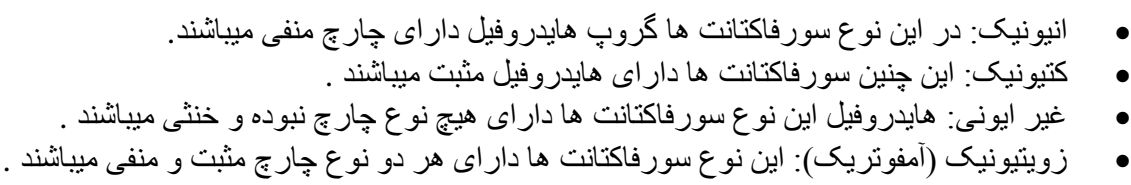

بخش هايدروفيليك سور فاكتانت ها تمايل بيشتر با فاز آبى دارند از همين لحاظ بنام آب دوست ياد ميشوند. اما، كروب: هايدروفوبيك سور فاكتانتانت ها مايل

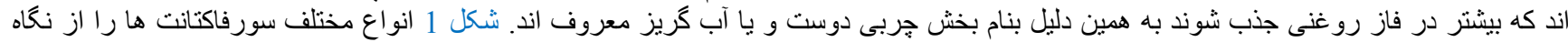

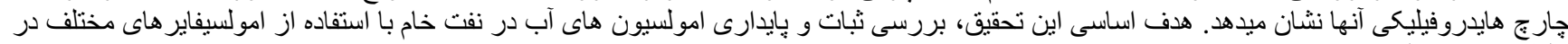
غلظت هارى مختلف ميباشثد.

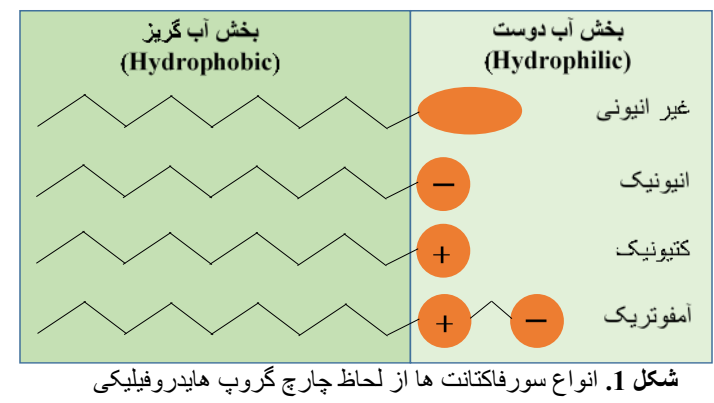

مواد

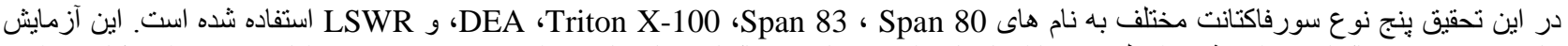

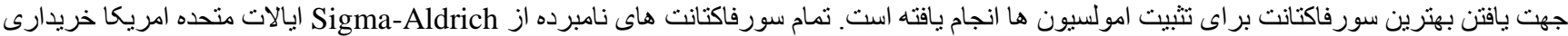

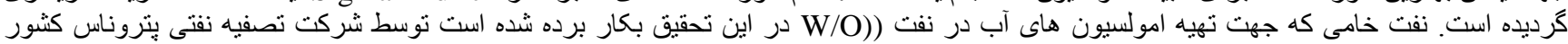




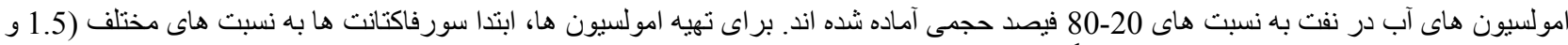

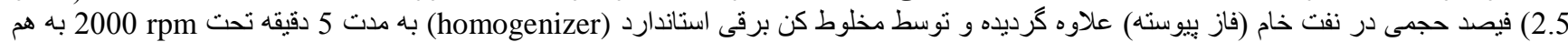

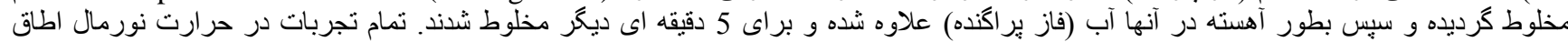

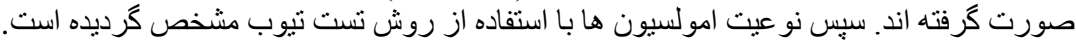

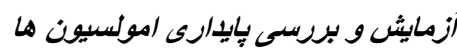

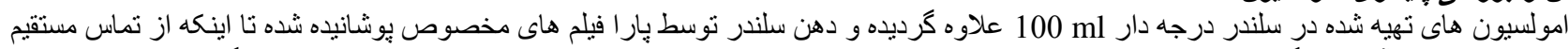

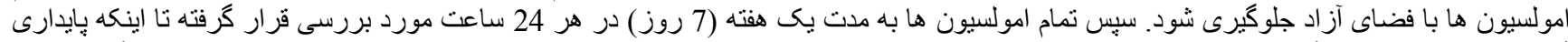

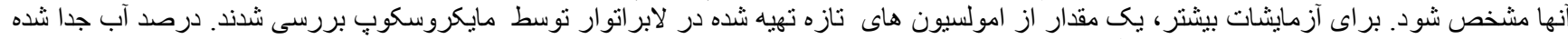

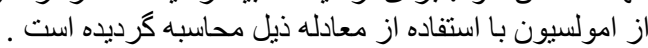

$$
\text { آب (ml) }
$$

تعيين اندازه قطرات /مولسبيون ها

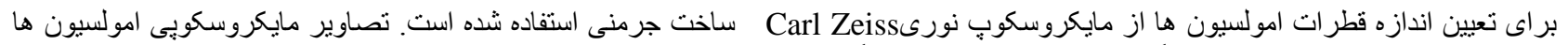

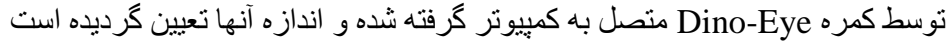

در اين آزمايشات، به اساس مشاهده بصرى، هيج يكى از امولسيون ها در دوره 7 روزه آب را جدا جدا نكرده و كاملا يايدار بودند. در كل، ثبات امولسيون

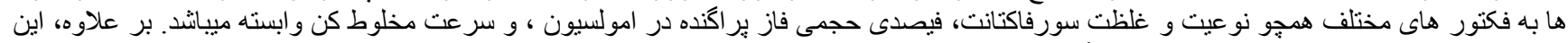

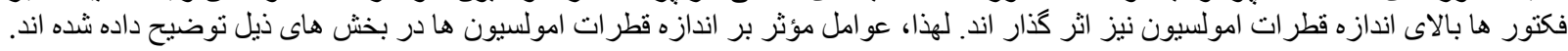

اثر نوعيت و غلظت امولسبفاير بر اندازه ق قطرات در /مولسبيون

به اساس اين تحقيق جنين دريافت كرديد كه نوعيت و غلظت سورفاكتانت بك عامل مؤثر بر اندازه قطر ات امولسيون ها بوده است. اساساً، در اين

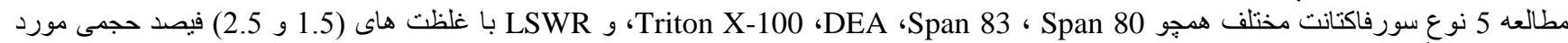

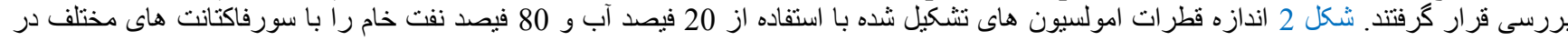
غلظت هاى مختلف نشان ميدهد.

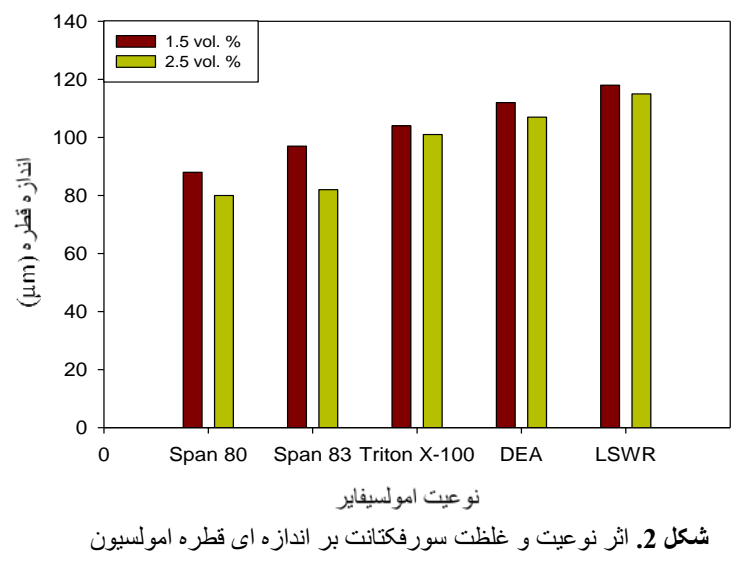

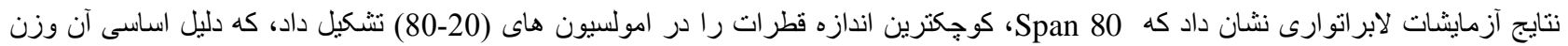

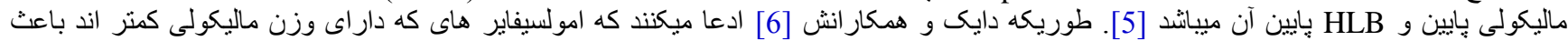

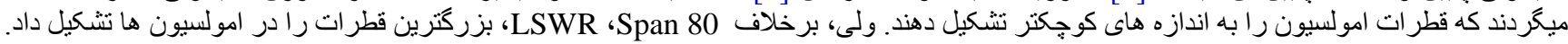

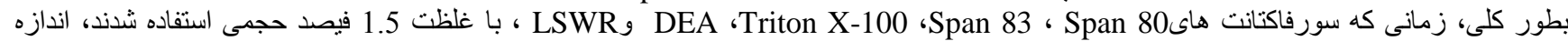

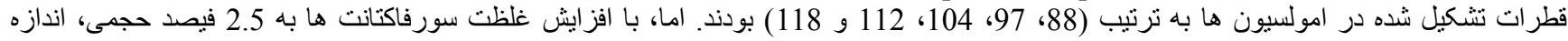

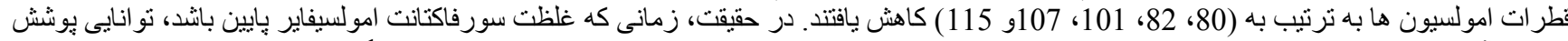

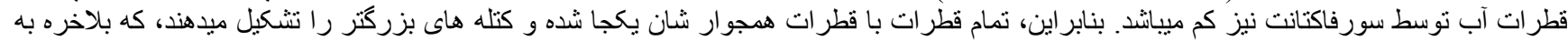

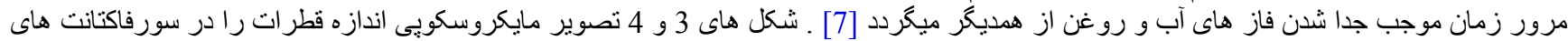




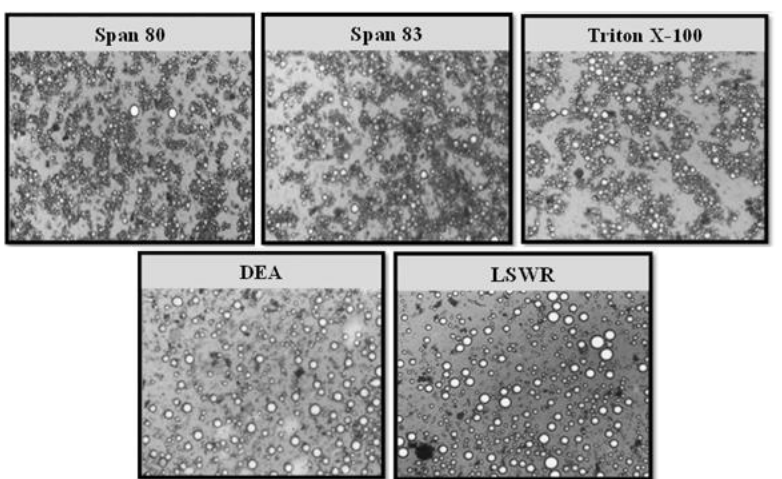

شكل 3. تصوير مايكروسكوب نورى امولسيون 20-80 فيصد آب در نفت تثييت شده توسط 1.5 فيصد حجمى ايمولسيفاير.
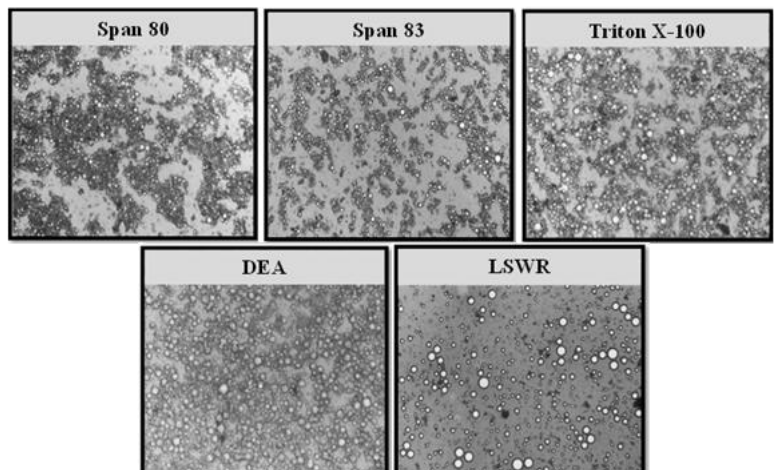

شكل 4. تصوير مايكروسكوب نورى امولسيون 80-20 فيصد آب در نفت تثيبت شده توسط 2.5 فيصد حجمى ايمولسيفاير.

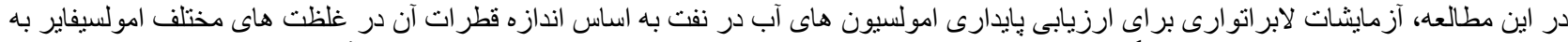

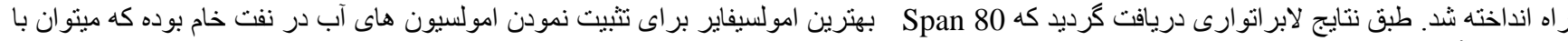

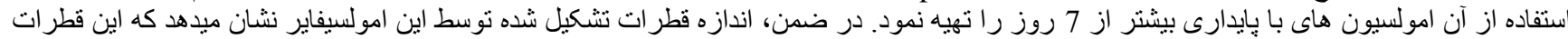

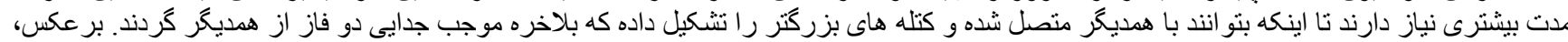

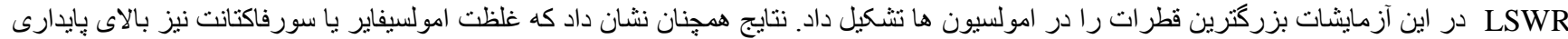

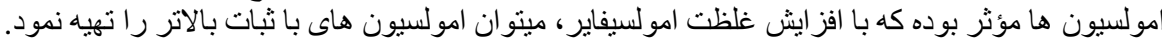

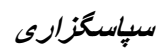

نويسندكان اين مقاله از يو هنتون يهنح ماليزيا جهت ههكارى و حمايت مالى براى تهيه نمودن مواد تحقيقى و ديكر بروسه هاى آزمايشى سباسكز ارى مى نمايند.

\section{References}

[1] K.K. Salam, a. O. Alade, a. O. Arinkoola, A. Opawale, Improving the Demulsification Process of Heavy Crude Oil Emulsion through Blending with Diluent, J. Pet. Eng. (2013) 1-6. doi:10.1155/2013/793101.

[2] L. Xia, S. Lu, G. Cao, Stability and demulsification of emulsions stabilized by asphaltenes or resins., J. Colloid Interface Sci. 271 (2004) 504-506. doi:10.1016/j.jcis.2003.11.027.

[3] E.J. Windhab, M. Dressler, K. Feigl, P. Fischer, D. Megias-Alguacil, Emulsion processing-from single-drop deformation to design of complex processes and products, Chem. Eng. Sci. 60 (2005) 2101-2113. doi:10.1016/j.ces.2004.12.003.

[4] M.J. Lawrence, G.D. Rees, Microemulsion-based media as novel drug delivery systems, Adv. Drug Deliv. Rev. 45 (2000) 89 121.

[5] F. Muzaffar, U.K. Singh, L. Chauhan, Review on microemulsion as futuristic drug delivery, Int. J. Pharm. Pharm. Sci. 5 (2013) 39-53.

[6] R. Daik, S. Bidol, I. Abdullah, Effect of molecular weight on the droplet size and rheological properties of liquid natural rubber emulsion, Malaysian Polym. J. 2 (2007) 29-38.

[7] S. Akbari, N.H. Abdurahman, F. Fayaz, The influence of process parameters on stability of water-in-crude oil emulsion stabilized by span 80, Int. J. Eng. Sci. Res. Technol. 4 (2015) 526-534. http://www.ijesrt.com/issues pdf file/Archives2015/May-2015/73. 